\title{
Stereotactic Body Radiation Therapy for Patients with Early-stage Prostate Cancer
}

\author{
HANNAH LINNEY and SARAH BARRETT \\ Applied Radiation Therapy Trinity, Discipline of Radiation Therapy, \\ School of Medicine, Trinity College, Dublin, Ireland
}

\begin{abstract}
Aim: Stereotactic body radiation therapy (SBRT) is emerging as a new treatment option for early-stage prostate cancer, theoretically providing clinical and economic benefits compared to conventionally fractionated external-beam radiation therapy (CF-EBRT). This review aimed to evaluate available published data to determine if the proposed theoretical benefits translate clinically. Materials and Methods: A systematic search strategy was employed across three databases using predefined search terms, inclusion and exclusion criteria to identify relevant articles. Results: Sixteen articles were included. Biochemical progression-free survival rates of 77.1-100\% were reported in SBRT studies compared to 55-98\% in CF-EBRT studies. Incidence of acute grade 1, 2, and 3 genitourinary toxicities were reported in the range of $13.3-71 \%, 12-25 \%$ and $0-3 \%$, respectively, in the SBRT cohort in comparison to $28.7-$ $51.9 \%, 15.6-41.4 \%$. and $1.1-8.1 \%$, respectively, in the CFEBRT cohort. Incidence of acute grade 1, 2, and 3 gastrointestinal toxicities were reported in the range of 13 $67 \%, 1-27 \%$ and $0-9 \%$, respectively, of the SBRT cohort compared to $16.1-51.1 \%, 6.3-20.7 \%$ and $0-3 \%$, respectively, of the CF-EBRT cohort. Mean treatment costs estimates associated with SBRT ranged from $\$ 22,152$ to $\$ 24,873$ and $\$ 33,068$ to $\$ 35,431$ for CF-EBRT. Conclusion: Available data support the hypothesis of lower rates of acute toxicity and reduced economic burden associated with SBRT compared to CF-EBRT, however, randomised data with
\end{abstract}

This article is freely accessible online.

Correspondence to: Sarah Barrett, Discipline of Radiation Therapy, Trinity Centre for Health Sciences, St. James's Hospital, Dublin 8, James's Street, Ushers, Dublin, Ireland. Tel: +353 8963248, e-mail: barrets7@tcd.ie

Key Words: Prostate cancer, stereotactic, SBRT, review. longer follow-up are needed to determine whether SBRT is clinically more effective than CF-EBRT.

Prostate cancer is the second most common malignancy in males, with increasing numbers of patients being diagnosed with early-stage disease $(1,2)$. Current potentially curative therapeutic options for these patients include conventionally fractionated external-beam radiation therapy (CF-EBRT), radical prostatectomy and brachytherapy (3). Stereotactic body radiation therapy (SBRT) is emerging as a new treatment option (4).

Current treatment options are considered comparable in terms of 5-year biochemical progression-free survival (bPFS) (3). CF-EBRT is a popular option, chosen by up to $46 \%$ of this patient group (5), however, longer treatment durations can be problematic for some patients (5). CF-EBRT involves delivery of a 1.8-2.0 Gy fraction, five days per week for seven to eight weeks to total doses ranging 78-81 Gy (6). SBRT is delivered in higher doses per fraction, ranging from 3.5 to $15 \mathrm{~Gy}$ in up to five fractions, and to total doses ranging from 30 to $50 \mathrm{~Gy}$ (6). This may be more convenient for patients and may also reduce the resource burden on departments. In 2009, treatment of prostate cancer cost $€ 81$ million in Ireland and $€ 116.7$ million in the UK in the first year since diagnosis $(1,7)$. Implementation of SBRT as a treatment option could potentially reduce this, increase patient throughput and reduce possible waiting lists.

SBRT also theoretically improves the therapeutic window for these patients, compared to CF-EBRT (8). In theory, an increased dose per fraction improves the tumour control probability of early-stage prostate tumours without increasing the normal tissue complication probability (NTCP) of the rectum and genitourinary (GU) tract, based on their associated $\alpha / \beta$ ratios (4), which is a radiobiological parameter that explains a tissue's behaviour with respect to a radiation schedule (6). A low $\alpha / \beta$ ratio can imply greater sensitivity to higher dose fractions, while higher $\alpha / \beta$ ratios suggest higher 
sensitivity to overall dose (9). Multiple publications suggest that early-stage prostate tumours have a low $\alpha / \beta$ ratio of 1.5 , implying greater tumour control probability with increased dose per fraction (9-11), while that of the rectum is estimated at 4-6 (12) and the $\alpha / \beta$ ratio of the GU tract is estimated at 3-7 (13). As the total dose of SBRT schemes is significantly less than that of CF-EBRT schemes, the NTCP of the rectum and GU tract could potentially be reduced.

Despite these proposed theoretical benefits, physicians are hesitant to adopt this treatment approach for this patient group. There is a lack of certainty that the linear-quadratic model of cell kill, from which the concept of $\alpha / \beta$ ratios is derived, may be applied to estimating dose-response with very large doses per fraction (4). This study aimed to address these concerns through analysis and comparison of clinical data of SBRT treatment for early-stage prostate cancer to CF-EBRT data.

\section{Materials and Methods}

A systematic search was performed across three electronic databases (Science direct, Cinahl and Pubmed) for studies of patients with early-stage prostate cancer treated with SBRT or CF-EBRT. Prospective and retrospective trials published between January 2007 and November 2016, with 40 or more participants were included. Studies reporting rates of low- to high-grade gastro-intestinal (GI) and GU toxicities using the Common Terminology Criteria for Adverse Events (14) or Radiation Therapy Oncology Group (15) scales were included. No published randomised control trials comparing the two interventions were identified. Ten SBRT studies reporting bPFS rates were included and seven SBRT studies reporting rates of acute low- to high-grade GI and GU toxicities. Four CF-EBRT studies reporting bPFS rates were identified and four CF-EBRT studies reporting crude rates of acute low- to highgrade GI and GU toxicities. Two studies using the Markov design analysis model to compare estimated mean costs of the two treatment options were included. The Downs and Black scale was used to assess quality of the studies collected (16).

\section{Results}

The primary database search yielded 696 results; 657 articles did not meet the inclusion criteria and were omitted based on title and abstract review. A secondary manual search of reference lists from primary articles, identified a further five articles for inclusion. Following a full text review, 18 studies fit the inclusion criteria. See Figure 1 for details.

Treatment efficacy. Outcome data from eight SBRT studies of patients with early-stage prostate cancer with a combined patient cohort of $n=2007$ were included (17-24). This included 1,281 low-risk, 622 intermediate-risk and 104 highrisk patients. Rates of bPFS reported ranged from $77.1 \%$ to $100 \%$ (17-24), at endpoints ranging 2-7 years post treatment.

Four studies reporting outcome data for early stage prostate cancer treated with CF-EBRT with a combined patient cohort of $n=1778$ were included in this review (27-30). This cohort consisted of 407 low-risk, 1,111 intermediate-risk and 254 high-risk patients. Reported rates of bPFS ranged from $55 \%$ to $98 \%$, at endpoints ranging 5-10 years post treatment.

Treatment toxicity. Rates of grade1 GU toxicity reported in SBRT studies $(n=1048)$ ranged from 13.3 to $71 \%(17,19-$ 23,25 ), grade 2 from 12 to $25 \%$ and grade 3 from 0 to $4 \%$. No grade 4 or higher acute GU toxicities were reported.

Rates of grade1 GU toxicity reported in CF-EBRT $(n=1118)$ studies ranged from 28.7 to $51.9 \%$ (30-32), grade 2 from 15.6 to $41.4 \%$ and grade 3 from 1.1 to $8 \%$. Two grade 4 acute GU toxicities were reported, a rate of $0.001 \%$ in the overall cohort. One CF-EBRT study $(\mathrm{n}=1065)$ neglected to stratify rates of GU toxicity by grade; it reported that $46 \%$ of patients treated experienced grade 2 or higher acute GU toxicity (29).

Rates of grade 1 GI toxicity reported in SBRT studies $(n=1048)$ were13-67\% $(17,19-23,25)$, grade 2 between 1 and $27 \%$, and grade 3 from 0 to $3 \%$.

Rates of grade1 GI toxicities reported in CF-EBRT studies ranged from 16.1 to $51.1 \%(30-32)$, grade 2 from 6.3 to $20.7 \%$ and grade 3 from 0 to $9 \%$. One CF-EBRT study neglected to stratify rates of acute GI toxicity by grade (29); it reported that $25 \%$ of patients treated EBRT experienced grade 2 or higher GI toxicity.

Two out of the four CF-EBRT studies that stated rates of toxicity $(n=965)$ reported interruption of treatment due to severe toxicity in 11 patients $(31,32)$. No SBRT study reported treatment interruption due to incidence of toxicity. Findings from studies on efficacy and toxicity are summarised in Tables I and II.

Treatment cost effectiveness. Two studies were identified that compared estimated mean costs of SBRT and CF-EBRT for early-stage prostate cancer $(33,34)$. Results from both studies support the hypothesis that SBRT is associated with a smaller economic burden for patients and departments compared to CF-EBRT. One study estimated the mean cost of SBRT treatment for patients with early-stage prostate cancer to be $\$ 22,152$ compared to $\$ 35,431$ for CF-EBRT (33). The other study estimated a mean lifetime cost of $\$ 24,873$ for patients treated with SBRT compared to $\$ 33,068$ for CF-EBRT (34). One study estimated that if $50 \%$ of patients with early-stage prostate cancer in the USA chose this option, annual societal savings of approximately $\$ 250$ million could be expected (33). No articles were found within the search that discredited this argument.

\section{Discussion}

The outcome data presented suggest at least equivalent efficacy of SBRT to CF-EBRT. However, the ability to directly compare outcomes and therefore definitively 


\section{Primary Search:}

Data bases: "Pubmed", "Science Direct" and "Cinahl" "prostate cancer"(Title/Abstract) NOT metastatic NOT "locally advanced" NOT recurrent AND radiotherapy(Title/Abstract) NOT "quality of life" NOT "dosimetric" NOT "planning" OR "prostate stereotactic"(keyword) OR “prostate SBRT”(keyword). 696 results

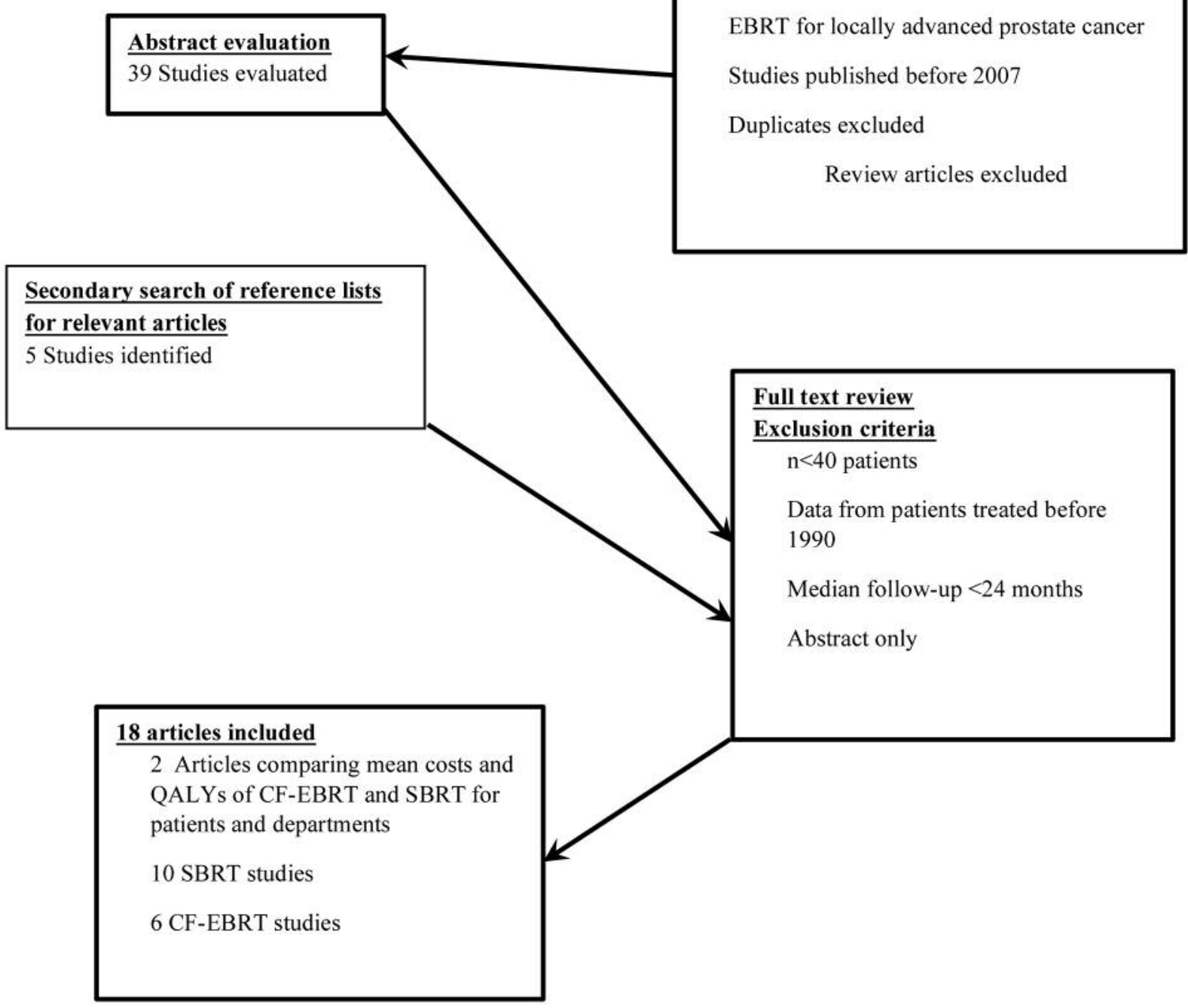

Figure 1. Database search strategy and results. SBRT: Stereotactic body radiation therapy; EBRT: external beam radiation therapy, QALYs: qualityadjusted life years; CF-EBRT: conventionally fractionated external beam radiation therapy.

determine if SBRT is more effective is limited. bPFS rates in SBRT studies are reported at varying endpoints, therefore they cannot be directly compared. Five out of the eight SBRT trials $(n=1355)$ reported bPFS at less than 5 years of follow-

\section{Exclusion criteria:}

Non-English language studies

Non-human studies

SBRT as a boost to complement EBRT

Non prostate cancer studies

SBRT for oligometastases

EBRT for recurrent prostate cancer

EBRT for locally advanced prostate cancer

sublished before 2007

Review articles excluded up $(19,20,23-25)$, while it was reported at a minimum of 5 years post treatment in all CF-EBRT studies (27-30).

In studies where rates of bPFS reported were stratified according to risk status, bPFS appears equivalent for both 
Table I. Summary of results from stereotactic body radiation therapy trials included.

\begin{tabular}{|c|c|c|c|c|c|c|c|c|c|c|}
\hline $\begin{array}{l}\text { Author } \\
\text { (Institution) }\end{array}$ & Analysis & $\begin{array}{l}\text { Patient } \\
\text { cohort }\end{array}$ & $\begin{array}{c}\text { Year } \\
\text { published }\end{array}$ & d $\begin{array}{c}\text { Dose } \\
\text { schedule } \\
\text { (delivery } \\
\text { method) }\end{array}$ & ADT & $\begin{array}{c}\text { Follow-up } \\
\text { (median) }\end{array}$ & $\begin{array}{c}\text { bPFS } \\
\text { (relapse= } \\
\text { nadir+2NG- } \\
\text { Phoenix } \\
\text { definition) }\end{array}$ & $\begin{array}{l}\text { Acute } \\
\text { urinary } \\
\text { toxicity }\end{array}$ & $\begin{array}{l}\text { Acute } \\
\text { rectal } \\
\text { toxicity }\end{array}$ & $\begin{array}{c}\text { Study quality } \\
\text { (using } \\
\text { Downs } \\
\text { Scale-max } \\
\text { score 26) }\end{array}$ \\
\hline $\begin{array}{l}\text { Bolzicco et al. } \\
\text { (17) } \\
\text { (San Bortolo } \\
\text { Hospital, } \\
\text { Vicenza, Italy) }\end{array}$ & Phase I/II & $\begin{array}{c}\mathrm{n}=100 \\
\text { Low risk, } \\
\mathrm{n}=41 \\
\text { Intermediate } \\
\text { risk, } \mathrm{n}=42 \\
\text { High risk, } \\
\mathrm{n}=17\end{array}$ & 2013 & $\begin{array}{l}35 \text { Gy } \\
\text { in } 5 \\
\text { fractions } \\
\text { daily } \\
\text { (CK) }\end{array}$ & $29 \%$ & $\begin{array}{c}36 \text { Months } \\
\text { (range=6-76 } \\
\text { months) }\end{array}$ & $\begin{array}{l}\text { (Included } \\
\text { in Kings } \\
\text { pooled } \\
\text { analysis } \\
\text { of } \\
\text { outcomes) }\end{array}$ & $\begin{array}{c}\text { RTOG: } \\
\text { G1, } n=34 \\
(34 \%) \\
\text { G2, } n=12 \\
(12 \%) \\
\text { G3+, } 0\end{array}$ & $\begin{array}{c}\text { RTOG: G1, } \\
\text { n=27 (27\%) } \\
\text { G2, } n=18 \\
(18 \%) \\
\text { G3+n=0\% }\end{array}$ & 14 \\
\hline $\begin{array}{l}\text { Loblaw et al. } \\
\text { (21) } \\
\text { (Sunnybrook, } \\
\text { Canada) }\end{array}$ & Phase I/II & $\begin{array}{c}\mathrm{n}=84 \\
\text { Low risk }\end{array}$ & 2013 & $\begin{array}{c}35 \text { Gy } \\
\text { in } 5 \\
\text { fractions } \\
\text { Once weekly } \\
\text { over } 29 \text { days } \\
\text { (IMRT) }\end{array}$ & $1 \%$ & $\begin{array}{c}55 \text { Months } \\
\text { (range }=13-68 \\
\text { months) }\end{array}$ & $\begin{array}{c}98 \% \\
@ 5 \text { years } \\
\text { (95\% CI= } \\
\text { 96-100\%) } \\
\text { One patient } \\
\text { experienced } \\
\text { biochemical } \\
\text { failure }\end{array}$ & $\begin{array}{c}\text { CTCAE: } \\
\text { G1, n=60 } \\
(71 \%) \\
\text { G2, n=4 } \\
(17 \%) \\
\text { G3, n=1 } \\
(1 \%)\end{array}$ & $\begin{array}{c}\text { CTCAE: G1, } \\
\text { n=56 }(67 \%) \\
\text { G2, } \mathrm{n}=8 \\
(10 \%) \\
\text { G3, } 0 \%\end{array}$ & 14 \\
\hline $\begin{array}{l}\text { Freeman et al. } \\
(25) \\
\text { (Naples, } \\
\text { Florida) }\end{array}$ & Phase I/II & $\begin{array}{c}n=41 \\
\text { Low risk }\end{array}$ & 2011 & $\begin{array}{l}35 \mathrm{~Gy} \\
\text { in } 5 \\
\text { fractions } \\
36.25 \mathrm{~Gy} \\
\text { in } 5 \\
\text { fractions } \\
\text { (CK) }\end{array}$ & $0 \%$ & $\begin{array}{c}60 \text { months } \\
\text { (range }=4.2- \\
6.2 \text { years })\end{array}$ & $\begin{array}{l}\text { (Included in } \\
\text { Kings pooled } \\
\text { analysis of } \\
\text { outcomes) }\end{array}$ & $\begin{array}{c}\text { RTOG: } \\
\text { G1, n=10 } \\
(25 \%) \\
\text { G2, n=3 } \\
(7 \%) \\
\text { G3, n=1 } \\
(2.5 \%)\end{array}$ & $\begin{array}{c}\mathrm{G} 1,13 \% \\
(6 / 41) \\
\mathrm{G} 2,2.5 \% \\
(1 / 41) \\
\mathrm{G} 3,0 \%\end{array}$ & 13 \\
\hline $\begin{array}{l}\text { Madsen } \text { et al. } \\
\text { (16) } \\
\text { (SHARP trial) } \\
\text { (VMMC- } \\
\text { Virginia } \\
\text { Mason } \\
\text { Medical } \\
\text { Centre) }\end{array}$ & Phase I/II & $\begin{array}{c}n=40 \\
\text { Low risk }\end{array}$ & 2007 & $\begin{array}{l}33.5 \mathrm{~Gy} \\
\text { in } 5 \text { daily } \\
\text { fractions } \\
\text { (3DCRT) }\end{array}$ & $0 \%$ & $\begin{array}{l}41 \text { months } \\
\text { (range }=12- \\
60 \text { months) }\end{array}$ & $\begin{array}{c}90 \% \\
\text { @ } 4 \text { years } \\
3 \text { patients } \\
\text { experienced } \\
\text { biochemical } \\
\text { failure (95\% } \\
\text { CI not } \\
\text { documented) }\end{array}$ & $\begin{array}{c}\mathrm{G} 1,11 \\
(28 \%) \\
\mathrm{G} 2,8 \\
(20.5 \%) \\
\mathrm{G} 3,1 \\
(2.5 \%)\end{array}$ & $\begin{array}{c}\mathrm{G} 1,10 \\
(26 \%) \\
\mathrm{G} 2,5(13 \%) \\
\mathrm{G} 3+, 0\end{array}$ & 14 \\
\hline $\begin{array}{l}\text { McBride et al. } \\
\text { (20) } \\
\text { (USA } \\
\text { multi- } \\
\text { institutional) }\end{array}$ & Phase I & $\begin{array}{c}n=45 \\
\text { Low risk }\end{array}$ & 2012 & $\begin{array}{c}36.25 \text { Gy in } \\
5 \text { fractions- } \\
34 \text { patients } \\
(76 \%) \\
37.5 \mathrm{~Gy} \text { in } \\
5 \text { fractions } \\
(22 \%) \\
1 \text { patient }(2 \%) \\
\text { dose not stated } \\
\text { Completed } \\
<10 \text { days } \\
(\mathrm{CK})\end{array}$ & )- & $\begin{array}{c}44.5 \text { months } \\
\text { (range }=0-62 \\
\text { months) }\end{array}$ & $\begin{array}{c}100 \% \\
@ 3 \text { years } \\
1 \text { patient } \\
\text { died of } \\
\text { unrelated } \\
\text { causes } \\
\text { there were no } \\
\text { biochemical } \\
\text { failures }\end{array}$ & $\begin{array}{c}\text { CTCAE: } \\
\text { G1, 25 } \\
(59 \%) \\
\text { G2, 819\%) } \\
\text { G3, 0\% } \\
42(93 \%) \\
\text { patients } \\
\text { were } \\
\text { available } \\
\text { for acute } \\
\text { toxicity } \\
\text { analysis }\end{array}$ & $\begin{array}{c}\text { CTCAE: } \\
\text { G1, 13/42 } \\
(31 \%) \\
\text { G2, 3/42 } \\
(27 \%) \\
\text { G3, } 0 \%\end{array}$ & 14 \\
\hline $\begin{array}{l}\text { King et al. } \\
(24) \\
\text { Pooled } \\
\text { analysis* }\end{array}$ & $\begin{array}{c}\text { Retro- } \\
\text { spective }\end{array}$ & $\begin{array}{c}\mathrm{n}=1,100 \\
\text { Low risk, } \\
\mathrm{n}=641 \\
\text { Intermediate } \\
\text { risk, } \mathrm{n}=334 \\
\text { high risk, } \\
\mathrm{n}=125\end{array}$ & 2013 & $\begin{array}{c}35 \mathrm{~Gy} \text { in } \\
5-\mathrm{n}=385 \\
36.25 \mathrm{~Gy} \\
\text { in } 5-\mathrm{n}=589 \\
38-40 \mathrm{~Gy} \\
\text { in } 5-\mathrm{n}=126 \\
\text { daily } \\
\text { (CK) }\end{array}$ & $\begin{array}{c}(14 \% \\
\text { of all } \\
\text { patients) } \\
\text { Low-50 } \\
(8 \%), \\
\text { Inter-49 } \\
(15 \%), \\
\text { High- } 48 \\
(38 \%)\end{array}$ & 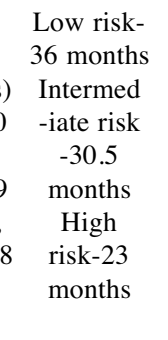 & $\begin{array}{c}94 \% \\
\text { @ } 3 \text { years } \\
\text { 5-year Kaplan- } \\
\text { Meier bPFS } \\
\text { Low risk, } \\
\text { 95.2\% } \\
\text { Intermediate } \\
\text { risk, 84.1\% } \\
\text { High risk, } \\
81.2 \%\end{array}$ & $\begin{array}{l}\text { Not } \\
\text { reported }\end{array}$ & $\begin{array}{c}\text { Not } \\
\text { reported }\end{array}$ & 17 \\
\hline $\begin{array}{l}\text { Chen et al. } \\
\text { (23) } \\
\text { (Georgetown } \\
\text { University) }\end{array}$ & $\begin{array}{c}\text { Retro- } \\
\text { spective }\end{array}$ & $\begin{array}{c}\mathrm{n}=100 \\
\text { Low risk, } \\
\mathrm{n}=37 \\
\text { Intermediate }\end{array}$ & 2014 & $\begin{array}{l}35 \text { Gy in } \\
5 \text { fractions } \\
(15 \%) \\
36.25 \text { Gy in }\end{array}$ & $11 \%$ & $\begin{array}{c}27.6 \text { months } \\
\text { (range }=1.4- \\
3.5 \text { years) }\end{array}$ & $\begin{array}{c}99 \% @ \\
2 \text { years } \\
\text { One } \\
\text { biochemical }\end{array}$ & $\begin{array}{c}\text { CTCAE: } \\
\text { G 1,36 } \\
(36 \%) \\
\text { G 2,35 }\end{array}$ & $\begin{array}{l}\text { CTCAE: } \\
\text { G } 1,20 \\
(20 \%) \\
\text { G } 2,1(1 \%)\end{array}$ & 15 \\
\hline
\end{tabular}


Table I. Continued

\begin{tabular}{|c|c|c|c|c|c|c|c|c|c|c|}
\hline $\begin{array}{l}\text { Author } \\
\text { (Institution) }\end{array}$ & Analysis & $\begin{array}{l}\text { Patient } \\
\text { cohort }\end{array}$ & $\begin{array}{c}\text { Year } \\
\text { published }\end{array}$ & $\begin{array}{c}\text { Dose } \\
\text { schedule } \\
\text { (delivery } \\
\text { method) }\end{array}$ & ADT & $\begin{array}{c}\text { Follow-up } \\
\text { (median) }\end{array}$ & $\begin{array}{c}\text { bPFS } \\
\text { (relapse= } \\
\text { nadir+2NG- } \\
\text { Phoenix } \\
\text { definition) }\end{array}$ & $\begin{array}{l}\text { Acute } \\
\text { urinary } \\
\text { toxicity }\end{array}$ & $\begin{array}{l}\text { Acute } \\
\text { rectal } \\
\text { toxicity }\end{array}$ & $\begin{array}{l}\text { Study quality } \\
\text { (using } \\
\text { Downs } \\
\text { Scale-max } \\
\text { score 26) }\end{array}$ \\
\hline & & $\begin{array}{c}\text { Risk, } \mathrm{n}=55 \\
\text { High risk, } \\
\mathrm{n}=8\end{array}$ & & $\begin{array}{l}5 \text { fractions } \\
(85 \%) \\
\text { alternate } \\
\text { days }(\mathrm{CK})\end{array}$ & & & $\begin{array}{c}\text { Failure in } \\
\text { a high risk } \\
\text { patient }\end{array}$ & $\begin{array}{l}(35 \%) \\
\text { G } 3,0\end{array}$ & G 3,0 & \\
\hline $\begin{array}{l}\text { Katz and } \\
\text { Kang (18) } \\
\text { (Winthrop } \\
\text { University } \\
\text { Hospital) }\end{array}$ & Phase I/II & $\begin{array}{c}\mathrm{n}=477 \\
\text { Low-risk, } \\
\mathrm{n}=324 \\
\text { Intermediate } \\
\text { risk, } \mathrm{n}=153\end{array}$ & 2013 & $\begin{array}{c}35 \text { Gy in } 5 \\
\text { fractions } n=50 \\
36.25 \text { Gy in } 5 \\
\text { fractions } n=427 \\
\text { daily }(\mathrm{CK})\end{array}$ & $19 \%$ & $\begin{array}{c}72 \text { months } \\
\text { (range }=0-96 \\
\text { months) }\end{array}$ & $\begin{array}{c}93.7 \% \\
\text { @ } 7 \text { years- } \\
\text { all patients } \\
\text { low risk- } \\
95.6 \% \\
\text { intermediate- } \\
\text { risk-89.6\% } \\
\text { @ } 7 \text { years } \\
11 \text { low-risk } \\
\text { and } 14 \\
\text { intermediate } \\
\text { risk } \\
\text { experienced } \\
\text { biochemical } \\
\text { failure }\end{array}$ & $\begin{array}{l}\text { RTOG: } \\
\text { G1, not } \\
\text { reported } \\
\text { G2, not } \\
\text { reported } \\
\text { G3+, } 0\end{array}$ & $\begin{array}{l}\text { RTOG: } \\
\text { G1, not } \\
\text { reported } \\
\text { G2, not } \\
\text { reported } \\
\text { G3+, } 0\end{array}$ & 14 \\
\hline $\begin{array}{l}\text { Oliai et al. (19) } \\
\text { (Drexel } \\
\text { University C } \\
\text { ollege, } \\
\text { Philadelphia) }\end{array}$ & $\begin{array}{c}\text { Retro- } \\
\text { spective }\end{array}$ & $\begin{array}{c}\mathrm{n}=70 \\
\text { Low risk, } \\
\mathrm{n}=36 \\
\text { Intermediate } \\
\text { risk, } \mathrm{n}=22 \\
\text { High risk, } \\
\mathrm{n}=12\end{array}$ & 2013 & $\begin{array}{c}35 \text { Gy in } \\
5 \text { fractions- } \\
n=5 \\
36.25 \text { Gy in } \\
5 \text { fractions- } \\
n=36 \\
37 \text { Gy in } \\
5 \text { fractions } \\
n=29 \\
\text { daily }(\mathrm{CK})\end{array}$ & $33 \%$ & $\begin{array}{c}31 \text { months } \\
\text { (range }=13-51 \\
\text { months) }\end{array}$ & $\begin{array}{c}\text { Low risk- } \\
100 \% \\
\text { Intermediate } \\
\text { risk-95\% } \\
\text { High risk- } \\
77.1 \% \\
\text { @ } 3 \text { years } \\
\text { 3-biochemical } \\
\text { failure }\end{array}$ & $\begin{array}{c}\text { RTOG: } \\
\text { G1, } 31(44 \%) \\
\text { G2, } 13(19 \%) \\
\text { G3, } 3(4 \%)\end{array}$ & $\begin{array}{c}\text { RTOG: } \\
\text { G1, } 7(17 \%) \\
\text { G2, } 6(9 \%) \\
\text { G3, } 2(3 \%)\end{array}$ & 13 \\
\hline $\begin{array}{l}\text { Hannan et al. } \\
(22) \\
\text { (USA-multi- } \\
\text { institutional) }\end{array}$ & $\begin{array}{l}\text { Phase } \mathrm{I} / \mathrm{II} \\
\text { Phase 1, } \\
47 \text { patients } \\
\text { Phase 2, } \\
\text { additional } \\
47 \text { patients }\end{array}$ & $\begin{array}{c}\mathrm{n}=91 \\
\text { Phase } 1 \\
45 \mathrm{~Gy} \\
\text { Low risk, } \\
\mathrm{n}=3 \\
\text { Intermediate } \\
\text { risk, } \mathrm{n}=12 \\
47.5 \mathrm{~Gy} \\
\text { Low- } \mathrm{n}=8 \\
\text { Intermediate } \\
\text { risk, } \mathrm{n}=7 \\
50 \mathrm{~Gy} \\
\text { Low risk, } \\
\mathrm{n}=7 \\
\text { Intermediate } \\
\text { risk, } \mathrm{n}=7 \\
\text { Phase } 2 \\
\text { Low risk, } \\
\mathrm{n}=15 \\
\text { Intermediate } \\
\text { risk, } \mathrm{n}=32\end{array}$ & 2016 & $\begin{array}{l}45 \text { Gy in } 5 \\
\text { fractions } \\
47.5 \text { Gy in } 5 \\
\text { fractions } \\
50 \text { Gy in } 5 \\
\text { fractions } \\
\text { (CK) }\end{array}$ & $16.5 \%$ & $\begin{array}{l}45 \text { Gy: } \\
74 \text { months } \\
47.5 \text { Gy: } \\
72 \text { months } \\
50 \text { Gy: } \\
66 \text { months }\end{array}$ & $\begin{array}{c}100 \% \\
\text { @ } 3 \text { years } \\
98.6 \% \\
\text { @ } 5 \text { years } \\
1 \text { intermediate } \\
\text {-risk patient } \\
\text { experienced } \\
\text { biochemical } \\
\text { failure } 40 \\
\text { months after } \\
\text { treatment }\end{array}$ & $\begin{array}{c}\text { CTCAE: } \\
\text { 45 Gy: } \\
\text { G1, } 2(13.3 \%) \\
\text { G2, } 5(33.3 \%) \\
\text { G3, } 0 \% \\
47.5 \text { Gy: } \\
\text { G1, } 8 \\
(53.3 \%) \\
\text { G2, } 1(6.7 \%) \\
\text { G3, } 0 \% \\
50 \text { Gy: } \\
\text { G1, 34 } \\
(55.7 \%) \\
\text { G2, } 14(23 \%) \\
\text { G3, } 0 \%\end{array}$ & $\begin{array}{c}\text { CTCAE: } \\
\text { 45 Gy: } \\
\text { G1, } 6(40 \%) \\
\text { G2, } 1(6.7 \%) \\
\text { G3, } 0 \% \\
47.5 \text { Gy: } \\
\text { G1, } 4 \\
(26.7 \%) \\
\text { G2, } 4 \\
(26.7 \%) \\
\text { G3, } 0 \% \\
50 \text { Gy: } \\
\text { G1, 34 } \\
(55.7 \%) \\
\text { G2, 14 } \\
(23 \%) \\
\text { G3, } 0 \%\end{array}$ & 15 \\
\hline
\end{tabular}

ADT: Androgen-deprivation therapy; bPFS: biochemical progression-free survival; RTOG: Radiation Therapy Oncology Group; IMRT: intensitymodulated radiation therapy; CK: Cyberknife; CTCAE: Common Terminology Criteria for Adverse Events; 3DCRT: 3D conformal radiotherapy; CI: confidence interval. *Participating institutions in the pooled analysis: Flushing Radiation Oncology, Flushing, NY, Naples Radiation Oncology, Naples, FL, Dept. of Radiation Oncology, Beth Israel Deaconness, Boston, MA, Radiosurgery Medical Group, San Diego, CA, Division of Radiation Oncology, San Bortolo Hospital, Vicenza, Italy, Dept. of Radiation Oncology, Stanford, CA, Dept. of Radiation Oncology, Georgetown University, Washington DC, Dept. of Radiation Oncology, Swedish Medical Center, Seattle, WA. **Participating institutions: multi-institutional trial: University of Texas Southwestern Medical Center, University of Minnesota, Prairie Lakes Hospital, University of Colorado, Orlando Health. American Urology Association. 
Table II. Summary of results from conventionally fractionated-external beam radiation therapy trials included.

\begin{tabular}{|c|c|c|c|c|c|c|c|c|c|c|}
\hline $\begin{array}{l}\text { Author } \\
\text { (Institution) }\end{array}$ & Analysis & $\begin{array}{l}\text { Patient } \\
\text { cohort }\end{array}$ & Year & $\begin{array}{c}\text { Dose } \\
\text { schedule }\end{array}$ & ADT & $\begin{array}{l}\text { Follow-up } \\
\text { (median) }\end{array}$ & $\begin{array}{c}\text { bRFS } \\
\text { (relapse= } \\
\text { nadir }+2 \text { NG- } \\
\text { Phoenix } \\
\text { definition) }\end{array}$ & $\begin{array}{l}\text { Acute } \\
\text { urinary } \\
\text { toxicity }\end{array}$ & $\begin{array}{l}\text { Acute } \\
\text { rectal } \\
\text { toxicity }\end{array}$ & $\begin{array}{c}\text { Study quality } \\
\text { (using } \\
\text { Downs } \\
\text { Scale, max } \\
\text { score 26) }\end{array}$ \\
\hline $\begin{array}{l}\text { Leborgne } \\
\text { et al. }(28) \\
\text { (Hospital } \\
\text { Italiano in } \\
\text { Montevideo, } \\
\text { Uruguay) }\end{array}$ & $\begin{array}{c}\text { Retro- } \\
\text { spective } \\
\text { (reporting } \\
\text { data from } \\
\text { conventional } \\
\text { dose } \\
\text { fractionation } \\
\text { arm) }\end{array}$ & $\begin{array}{c}\mathrm{n}=138 \\
\text { Low-risk, } \\
\mathrm{n}=59 \\
\text { Intermediate- } \\
\text { risk, } \\
\mathrm{n}=70 \\
\text { High-risk, } \\
\mathrm{n}=9\end{array}$ & 2009 & $\begin{array}{l}\text { median- } \\
78 \text { Gy } \\
\text { over } 55 \text { days } \\
\text { (3DCRT) } \\
\text { four-field box }\end{array}$ & $40 \%$ & $\begin{array}{c}49 \text { Months } \\
\text { (range, } 24-73 \\
\text { months) }\end{array}$ & $\begin{array}{c}\text { Low-risk, } \\
98 \% \\
(95 \% \mathrm{CI}= \\
96.9-99.5 \%) \\
\text { Intermediate- } \\
\text { risk, } 84 \% \\
(95 \% \mathrm{CI}= \\
70.8-98.5 \%) \\
\text { High-risk, 87\% } \\
(95 \% \mathrm{CI}= \\
74-99.9 \%) \\
\text { @ 5 years }\end{array}$ & $\begin{array}{c}\text { Not } \\
\text { reported }\end{array}$ & $\begin{array}{c}\text { Not } \\
\text { reported }\end{array}$ & 15 \\
\hline $\begin{array}{l}\text { Dearnaley } \\
\text { et al. }(26) \\
\text { (CHHiP trial) } \\
\text { international } \\
\text { multi-centre }\end{array}$ & $\begin{array}{c}\text { RCT } \\
\text { (reporting } \\
\text { data from } \\
\text { conventional } \\
\text { dose } \\
\text { fractionation } \\
\text { arm) }\end{array}$ & $\begin{array}{c}\mathrm{n}=1065 \\
\text { Low-risk, } \\
\mathrm{n}=157 \\
(15 \%) \\
\text { Intermediate- } \\
\text { risk, } \mathrm{n}=779 \\
(73 \%) \\
\text { High-risk, } \\
\mathrm{n}=129(12 \%)\end{array}$ & 2016 & $\begin{array}{c}74 \text { Gy in } \\
37 \text { fractions } \\
\text { daily } \\
\text { (IMRT) }\end{array}$ & $97 \%$ & $\begin{array}{l}62.6 \text { months } \\
\text { (54 months- } \\
77 \text { months) }\end{array}$ & $\begin{array}{c}88.3 \% \\
(95 \% \text { CI } \\
86.0-90.2) \\
@ 5 \text { Years } \\
\text { Low-risk, } \\
96.7 \%(95 \% \\
\text { CI=92.3-98.6) } \\
\text { Intermediate- } \\
\text { risk, 86.8\% } \\
(95 \% \mathrm{Cl}= \\
84.0-89.1) \\
\text { High risk-8 } \\
6.5 \%(95 \% \\
\text { CI-78.4-91.7) } \\
\text { 111/1065 had } \\
\text { biochemical } \\
\text { failure }\end{array}$ & $\begin{array}{c}\text { RTOG: } \\
\text { Grade 2+: } \\
\text { n=331 } \\
(46 \%)\end{array}$ & $\begin{array}{c}\text { RTOG: } \\
\text { Grade 2+: } \\
\text { n=176 } \\
(25 \%)\end{array}$ & 15 \\
\hline $\begin{array}{l}\text { Dearnaley } \\
\text { et al. } \\
\text { (27) } \\
\text { (MRC RT01 } \\
\text { trial } \\
\text { international } \\
\text { multi-centre) }\end{array}$ & $\begin{array}{c}\text { RCT } \\
\text { (reporting } \\
\text { data from } \\
\text { conventional } \\
\text { dose } \\
\text { fractionation } \\
\text { arm) }\end{array}$ & $\begin{array}{c}\mathrm{n}=422 \\
\text { Low-risk, } \\
\mathrm{n}=81(20 \%) \\
\text { Intermediate- } \\
\text { risk, } \mathrm{n}=152 \\
(36 \%) \\
\text { High-risk, } \\
\mathrm{n}=184(44 \%)\end{array}$ & 2014 & $\begin{array}{c}74 \text { Gy in } \\
37 \text { fractions } \\
\text { daily } \\
\text { (3DCRT) }\end{array}$ & $100 \%$ & 10 years & $\begin{array}{c}\text { 55\% (95\% } \\
\text { CI-50-61)@ } \\
10 \text { years } \\
\text { Approximately } \\
80 \% \text { @ 5years } \\
\text { (according to } \\
\text { Kaplan-Meier } \\
\text { graph, not } \\
\text { otherwise } \\
\text { documented) } \\
\text { 170/421 (43\%) } \\
\text { experienced } \\
\text { biochemical } \\
\text { progression }\end{array}$ & $\begin{array}{c}\text { Not } \\
\text { reported }\end{array}$ & $\begin{array}{c}\text { Not } \\
\text { reported }\end{array}$ & 14 \\
\hline $\begin{array}{l}\text { Beckendorf } \\
\text { et al. (29) } \\
\text { (GETUG } 06 \\
\text { trial multi- } \\
\text { centre } \\
\text { France) }\end{array}$ & $\begin{array}{c}\text { RCT } \\
\text { (reporting } \\
\text { data from } \\
\text { conventional } \\
\text { dose } \\
\text { fractionation } \\
\text { arm) }\end{array}$ & $\begin{array}{c}\mathrm{n}=153 \\
\text { Intermediate- } \\
\text { risk, } \mathrm{n}=110 \\
\text { High-risk, } \\
\mathrm{n}=43\end{array}$ & 2011 & $\begin{array}{l}80 \text { Gy in } \\
40 \text { fractions } \\
\text { (3DCRT) } \\
\text { daily }\end{array}$ & $0 \%$ & 61 Months & $\begin{array}{c}76.5 \% \\
\text { @ } 5 \text { years } \\
6 \text { Patients } \\
\text { died of } \\
\text { prostate } \\
\text { cancer by } \\
54 \text { months }\end{array}$ & $\begin{array}{c}\text { RTOG: } \\
\text { G1: } 32 \% \\
\text { G2: } 24 \% \\
\text { G3: } 3 \%\end{array}$ & $\begin{array}{l}\text { RTOG: } \\
\text { G1: } 34 \% \\
\text { G2: } 21 \% \\
\text { G3: } 9 \%\end{array}$ & 16 \\
\hline $\begin{array}{l}\text { Jereczek- } \\
\text { Fossa } \\
\text { et al. (30) }\end{array}$ & $\begin{array}{l}\text { Non- } \\
\text { randomised } \\
\text { prospective }\end{array}$ & $\begin{array}{c}\mathrm{n}=174 \\
\text { Low-risk, } \\
\mathrm{n}=32\end{array}$ & 2011 & $\begin{array}{c}80 \text { Gy in } \\
40 \\
\text { (3DCRT) }\end{array}$ & $69 \%$ & $\begin{array}{c}\text { Not } \\
\text { applicable }\end{array}$ & Not reported & $\begin{array}{c}\text { RTOG: } \\
\text { G1: } n=72 \\
(41.4 \%)\end{array}$ & $\begin{array}{c}\text { RTOG: } \\
\text { G1: } n=28 \\
(16.1 \%)\end{array}$ & 15 \\
\hline
\end{tabular}


Table II. Continued

\begin{tabular}{|c|c|c|c|c|c|c|c|c|c|c|}
\hline $\begin{array}{l}\text { Author } \\
\text { (Institution) }\end{array}$ & Analysis & $\begin{array}{l}\text { Patient } \\
\text { cohort }\end{array}$ & Year & $\begin{array}{c}\text { Dose } \\
\text { schedule }\end{array}$ & ADT & $\begin{array}{l}\text { Follow-up } \\
\text { (median) }\end{array}$ & $\begin{array}{c}\text { bRFS } \\
\text { (relapse= } \\
\text { nadir }+2 \text { NG- } \\
\text { Phoenix } \\
\text { definition) }\end{array}$ & $\begin{array}{l}\text { Acute } \\
\text { urinary } \\
\text { toxicity }\end{array}$ & $\begin{array}{l}\text { Acute } \\
\text { rectal } \\
\text { toxicity }\end{array}$ & $\begin{array}{c}\text { Study quality } \\
\text { (using } \\
\text { Downs } \\
\text { Scale, max } \\
\text { score 26) }\end{array}$ \\
\hline $\begin{array}{l}\text { (European } \\
\text { Institute of } \\
\text { Oncology, } \\
\text { Milan, Italy) }\end{array}$ & $\begin{array}{c}\text { Study } \\
\text { (reporting } \\
\text { data from } \\
\text { conventional } \\
\text { dose } \\
\text { fractionation } \\
\text { arm) }\end{array}$ & $\begin{array}{c}\text { Intermediate- } \\
\text { risk, } \mathrm{n}=68 \\
\text { High-risk } \\
\mathrm{n}=74 \\
\end{array}$ & & daily & & & & $\begin{array}{c}\mathrm{G} 2: \mathrm{n}=36 \\
(20.7 \%) \\
\mathrm{G} 3: \mathrm{n}=1 \\
(0.6 \%) \\
\mathrm{G} 4: \mathrm{n}=2 \\
(1.1 \%) \\
\text { (two patients } \\
\text { treatment } \\
\text { interrupted } \\
\text { due to toxicity) }\end{array}$ & $\begin{array}{c}\mathrm{G} 2: \mathrm{n}=11 \\
(6.3 \%) \\
\mathrm{G} 3+: 0 \%\end{array}$ & \\
\hline $\begin{array}{l}\text { Matzinger } \\
\text { et al. }(31) \\
\text { EORTC trial } \\
22991 \\
\text { multi- } \\
\text { institutional } \\
\text { international) }\end{array}$ & Retrospective & $\begin{array}{l}791 \\
\text { Intermediate- } \\
\text { and high-risk }\end{array}$ & 2009 & $\begin{array}{l}70 \text { Gy-78 } \\
\text { Gy in } 2 \\
\text { Gy/fraction } \\
\text { (3DCRT) } \\
74-78 \mathrm{~Gy} \\
\text { in } 2 \mathrm{~Gy} / \\
\text { fraction } \\
\text { (IMRT) } \\
\text { daily }\end{array}$ & $50 \%$ & 22 months & Not reported & $\begin{array}{c}\text { CTCAE: } \\
70 \text { Gy: } \\
\text { G1,n=91 } \\
(46.7 \%) \\
\text { G2, n=65 } \\
(33.3 \%) \\
\text { G3, n=14 } \\
(7.2 \%) \\
74 \text { Gy: } \\
\text { G1, n=195 } \\
(51.9 \%) \\
\text { G2, n=131 } \\
(34.8 \%) \\
\text { G3, n=18 } \\
(4.8 \%) \\
78 \text { Gy: } \\
\text { G1, n=37 } \\
(45.7 \%) \\
\text { G2, n=31 } \\
(38.3 \%) \\
\text { G3, n=6 } \\
(7.4 \%) \\
74 \text { Gy: IMRT } \\
\text { G1, n=13 } \\
(46.4 \%) \\
\text { G2, n=7 (25\%) } \\
\text { G3, n=3 } \\
(10.7 \%) \\
78 \text { Gy: IMRT } \\
\text { G1, 53/111 } \\
(47.7 \%) \\
\text { G2, 46/111 } \\
(41.4 \%) \\
\text { G3, 9/111 } \\
(8.1 \%) \\
(9 \text { patients } \\
\text { temporarily } \\
\text { interrupted } \\
\text { treatment due } \\
\text { to acute } \\
\text { toxicity) }\end{array}$ & 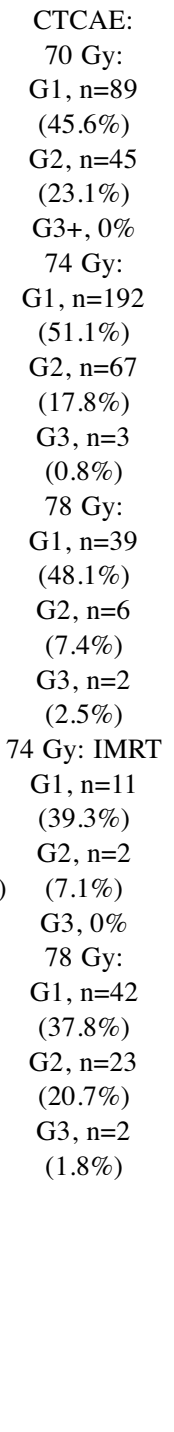 & 更 \\
\hline
\end{tabular}

ADT: Androgen-deprivation therapy; bPFS: biochemical progression-free survival; RTOG: Radiation Therapy Oncology Group; IMRT: intensitymodulated radiation therapy; CTCAE: Common Terminology Criteria for Adverse Events; 3DCRT: 3D conformal radiotherapy; CI: confidence interval; RCT: randomised controlled trial. 
treatment options among low-risk patients at 5 years of follow-up. However, among intermediate-risk patients, rates appear more favourable in the SBRT cohort than in the CFEBRT cohort. Further statistical analysis would be required to identify a definitive difference between the treatment options.

At 5 years of follow-up, there was a wider range of bPFS rates reported in CF-EBRT studies. The proportion of lowrisk patients included in SBRT trials (64\%) was found to be considerably higher than that in CF-EBRT trials (23\%). There was also a higher proportion of intermediate-risk (62\%) and high-risk (14\%) patients included in CF-EBRT trials compared to those treated with SBRT (31\% and 5\%, respectively). Risk classification stratifies patients according to specific tumour characteristics as a method of predicting outcome (35). It is expected that low-risk patients would experience greater disease control than intermediate- or highrisk patients (35), which may account for more favourable outcomes among the SBRT cohort.

Furthermore, the CF-EBRT studies reported on patients treated between 1990 and 2011 and the SBRT studies between 2002 and 2013. There may be a potential bias in terms of developments in imaging modalities used for staging, grading and treatment delivery during this time.

The quality of evidence supporting SBRT also limits the ability to determine whether it is more effective. Five out of the eight SBRT trials included were single institutional trials $(23,25,26,28,30)$. Their data is therefore not necessarily generalizable to the entire patient population. Sample sizes within SBRT trials were relatively small; six out of the eight SBRT trials reporting outcomes had 100 or fewer participants $(23,26-30)$. As sample size is inversely proportional to the margin of error, this is also a limiting factor (36). A single multi-national, multi-institutional pooled analysis of patients treated with SBRT $(n=1100)$ was identified, reporting rates of bPFS at 3 years that are consistent with results reported in other SBRT studies of poorer quality (25).

Toxicity data presented support the hypothesis that lower rates of toxicity are associated with SBRT compared to CFEBRT, in the acute setting. However, the highest rates of grade1 GI and GU toxicities in an individual study were reported in an SBRT trial (22). This is the only SBRT trial in which treatments were delivered using a standard linear accelerator using online megavoltage portal imaging matched to gold fiducials in the prostate for daily image guidance. In the other SBRT trials included, treatment was delivered using a Cyberknife. Cyberknife allows for precise tumour tracking to sub-millimetre accuracy, compared to millimetre accuracy of other linac-based systems (37). Greater confidence in precise dose delivery to the target volume allows a potential reduction of clinical target volume planning target volume (CTV-PTV) margins and potentially increased sparing of normal tissue achieved by steeper dose gradients around the PTV (5).
All four CF-EBRT studies included reported use of megavoltage portal imaging for image guidance. Imaging protocols ranged from weekly to once every 2 weeks, compared to daily online imaging in all SBRT trials. It is therefore difficult to determine if lower toxicity rates can be attributed to the theorised radiobiological parameters of the tumours and surrounding tissues or the more robust image guidance and imaging frequency reported in the SBRT trials.

Both studies that analysed cost effectiveness of the treatment options $(33,34)$ used the Markov design analysis model, a very well-recognised method of comparative effectiveness analysis (4). The factors affecting the Markov design analysis model are cost, quality of life and efficacy (bPFS) (4). Both studies assumed SBRT and CF-IMRT were equally effective and associated with equal rates of treatment-induced toxicity, based on the current data available for SBRT. However, given the lack of long-term follow-up, little is known about late effects that could impact negatively on patients' quality of life.

Further studies with longer follow-up are required for formal comparison of SBRT and CF-EBRT in order to definitively determine if SBRT is a more effective and less toxic treatment approach. A limiting factor of this review is that not all participants included received adjuvant androgen deprivation therapy (ADT). In SBRT trials, the number of patients prescribed ADT ranged from 0 to $38 \%$ of the given patient cohorts. In comparison, in CF-EBRT studies, 40$100 \%$ were prescribed ADT. Level one evidence shows significant improvement of bPFS when patients receive adjuvant ADT compared to RT alone (38). This further demonstrates the need for a randomised controlled trial comparing SBRT with CF-EBRT, within which use of ADT should be protocolled.

\section{Conclusion}

Available clinical data of early-stage prostate cancer treatment with SBRT suggest it to be an attractive alternative to CF-EBRT. Longer term follow-up, larger patient cohorts and randomised data are needed to allow direct comparison of the two techniques.

\section{Conflicts of Interest}

None.

\section{References}

1 Cancer in Ireland 1994-2013: Annual Report of the National Cancer Registry. NCR, Cork, Ireland.

2 Pashayan N, Pharoah P, Neal DE, Hamdy F, Donovan J, Martin RM, Greenberg D and Duffy SW: Stage shift in PSA detected prostate cancers - effect modification by Gleason score: Gleason score interaction with stage shift. J Med Screen 16(2): 98-101, 2009. 
3 Kupelian PA, Potters L, Khuntia D, Ciezki JP, Reddy CA, Reuther AM, Carlson, TP and Klein EA: Radical prostatectomy, external beam radiotherapy $<72 \mathrm{~Gy}$, external beam radiotherapy $>$ or $=72 \mathrm{~Gy}$, permanent seed implantation, or combined seeds/external beam radiotherapy for stage T1-T2 prostate cancer. Int J Radiat Oncol Biol Phys 58(1): 25-33, 2004.

4 Henderson DR, Tree AC and van As NJ: Overview: stereotactic body radiotherapy for prostate cancer. Clinical Oncol 27: 270279, 2015.

5 Tan T-J, Siva S, Foroudi F and Gill S: Stereotactic body radiotherapy for primary prostate cancer: A systematic review. J Med Imag Radiat Oncol 58(5): 601-611, 2014.

6 Zaorsky NG, Studenski MT, Dicker AP, Gomella L and Den RB: Hot Topic: Stereotactic body radiation therapy for prostate cancer: Is the technology ready to be the standard of care? Cancer Treat Rev 39: 212-218, 2013.

7 Fourcade R, Benedict Ã, Black L, Stokes M, Alcaraz A and Castro R: Treatment costs of prostate cancer in the first year after diagnosis: a short-term cost of illness study for France, Germany, Italy, Spain and the UK. BJU Int 105(1): 49-56, 2010

8 Moon DH, Efstathiou JA and Chen RC: What is the best way to radiate the prostate in 2016? Urol Oncol 35(2): 59-68, 2017.

9 Brenner D, Armour E, Corry P and Hall E: Sublethal damage repair times for a late-responding tissue relevant to brachytherapy (and external-beam radiotherapy): Implications for new brachytherapy protocols. Int J Radiat Oncol Biol Phys 41(1): 135-138, 1998.

10 Bentzen SM and Ritter MA: The alpha/beta ratio for prostate cancer: What is it, really? Radiother Oncol 76(1): 1-3, 2005.

11 Duchesne GM and Peters LJ: What is the alpha/beta ratio for prostate cancer? Rationale for hypofractionated high-dose-rate brachytherapy. Int J Radiat Oncol Biol Phys 44(4): 747-748, 1999.

12 van der Kogel AJ, Jarrett KA, Paciotti MA and Raju MR: Radiation tolerance of the rat rectum to fractionated X-rays and pi-mesons. Radiother Oncol 12(3): 225-232, 1988.

13 Cabrera AR and Lee WR: Hypofractionation for clinically localized prostate cancer. Semin Radiat Oncol 23: 191-197, 2013.

14 Trotti A, Byhardt R, Stetz J, Gwede C, Corn B, Fu K, Gunderson L, McCormick B, Morris M, Rich T and Shipley W: Common toxicity criteria: version 2.0. an improved reference for grading the acute effects of cancer treatment: impact on radiotherapy. Int J Radiat Oncol Biol Phys 47(1): 13-47, 2000.

15 Cox JD, Stetz J and Pajak TF: Toxicity criteria of the Radiation Therapy Oncology Group (RTOG) and the European organization for research and treatment of cancer (EORTC). Int J Radiat Oncol Biol Phys 31(5): 1341-1346, 1995.

16 Downs SH and Black N: The feasibility of creating a checklist for the assessment of the methodological quality both of randomised and non-randomised studies of health care interventions. J Epidemiol Community Health 52(6): 377-384, 1998.

17 Madsen BL, Hsi RA, Pham HT, Fowler JF, Esagui L and Corman J: Stereotactic hypofractionated accurate radiotherapy of the prostate (SHARP), 33.5Gy in five fractions for localized disease: first clinical trial results. Int J Radiat Oncol Biol Phys 67(4): 1099-105, 2007.

18 Katz AJ and Kang J: Quality of life and toxicity after SBRT for organ-confined Prostate Cancer, a 7-Year Study. Front Oncol 4: 301,2014
19 Oliai C, Lanciano R, Sprandio B, Yang J, Lamond J, Arrigo S, Good M, Mooreville M, Garber B and Brady LW: Stereotactic body radiation therapy for the primary treatment of localized prostate cancer. J Radiat Oncol 2(1): 63-70, 2013.

20 McBride SM, Wong DS, Dombrowski JJ, Harkins B, Tapella P, Hanscom HN, Collins SP and Kaplan ID: Hypofractionated stereotactic body radiotherapy in low-risk prostate adenocarcinoma: preliminary results of a multi-institutional phase 1 feasibility trial. Cancer 118(15): 3681-3690, 2012.

21 Loblaw A, Cheung P, D'Alimonte L, Deabreu A, Mamedov A, Zhang L, Tang, C, Quon H, Jain S, Pang G and Nam R: Prostate radiotherapy: Prostate stereotactic ablative body radiotherapy using a standard linear accelerator: Toxicity, biochemical, and pathological outcomes. Radiother Oncol 107: 153-158, 2013.

22 Hannan R, Tumati V, Xie XJ, Cho LC, Kavanagh BD, Brindle J, Raben D, Nanda A, Cooley S, Kim DN and Pistenmaa, D: Stereotactic body radiation therapy for low and intermediate risk prostate cancer-Results from a multi-institutional clinical trial. Eur J Cancer 59: 142-151, 2016.

23 Chen LN, Suy S, Wang H, Bhagat A, Woo JA, Moures RA, Kim JS, Yung TM, Lei S, Collins BT and Kowalczyk K: Patientreported urinary incontinence following stereotactic body radiation therapy (SBRT) for clinically localized prostate cancer. Radiat Oncol 9: 148, 2014.

24 Bolzicco G, Favretto MS, Satariano N, Scremin E, Tambone C and Tasca A: A single-center study of 100 consecutive patients with localized prostate cancer treated with stereotactic body radiotherapy. BMC Urol 13(1): 49, 2013.

25 King CR, Freeman D, Kaplan I, Fuller D, Bolzicco G, Collins S, Meier R, Wang J, Kupelian P, Steinberg M and Katz A: Stereotactic body radiotherapy for localized prostate cancer: Pooled analysis from a multi-institutional consortium of prospective phase II trials. Radiotherapy and Oncology 109: 217-221, 2013.

26 Freeman DE and King CR: Stereotactic body radiotherapy for low-risk prostate cancer: five-year outcomes. Radiat Oncol 6(1): $1-5,2011$.

27 Dearnaley D, Syndikus I, Mossop H, Khoo V, Birtle A, Bloomfield D, Graham J, Kirkbride P, Logue J, Malik Z and Money-Kyrle J: Conventional versus hypofractionated high-dose intensity-modulated radiotherapy for prostate cancer: 5-year outcomes of the randomised, non-inferiority, phase $3 \mathrm{CHHiP}$ trial. Lancet Oncol 17(8): 1047-1060, 2016.

28 Beckendorf V, Guerif S, Le Prisé E, Cosset J-M, Bougnoux A, Chauvet B, Salem N, Chapet O, Bourdain S, Bachaud JM and Maingon P: 70Gy Versus 80Gy in localized prostate cancer: 5year results of GETUG 06 Randomized Trial. Int J Radiat Oncol Biol Phys 80(4): 1056-1063, 2011.

29 Dearnaley DP, Jovic G, Syndikus I, Khoo V, Cowan RA, Graham JD, Aird EG, Bottomley D, Huddart RA, Jose CC and Matthews JH: Articles: Escalated-dose versus control-dose conformal radiotherapy for prostate cancer: long-term results from the MRC RT01 randomised controlled trial. Lancet Oncol 15(4): 464-473, 2014.

30 Leborgne F and Fowler J: Clinical investigation: Late outcomes following hypofractionated conformal radiotherapy $v s$. standard fractionation for localized prostate cancer: a nonrandomized contemporary comparison. Int J Radiat Oncol Biol Phys 74: 1441-1446, 2009. 
31 Jereczek-Fossa BA, Zerini D, Fodor C, Santoro L, Cambria R, Garibaldi C, Tagaste B, Vavassori A, Cattani F, Alterio D and Gherardi F: Original article: Acute toxicity of image-guided hypofractionated radiotherapy for prostate cancer: Nonrandomized comparison with conventional fractionation. Urol Oncol 29: 523532,2011

32 Matzinger O, Duclos F, Bergh Avd, Carrie C, Villà S, Kitsios P, Poortmans P, Sundar S, van der Steen-Banasik EM, Gulyban A and Collette L: Acute toxicity of curative radiotherapy for intermediate- and high-risk localised prostate cancer in the EORTC trial 22991. Eur J Cancer 45: 2825-2834, 2009.

33 Hodges JC, Lotan Y, Boike TP, Benton R, Barrier A and Timmerman RD: Cost-effectiveness analysis of stereotactic body radiation therapy versus intensity-modulated radiation therapy: an emerging initial radiation treatment option for organ-confined prostate cancer. J Oncol Pract 8(3 Suppl): e31s-7s, 2012.

34 Parthan A, Pruttivarasin N, Davies D, Taylor DC, Pawar V, Bijlani A, Lich $\mathrm{KH}$ and Chen RC: Comparative costeffectiveness of stereotactic body radiation therapy versus intensity-modulated and proton radiation therapy for localized prostate cancer. Front Oncol 2: 81, 2012.

35 Rodrigues G, Warde P, Pickles T, Crook J, Brundage M, Souhami L and Lukka H: Pre-treatment risk stratification of prostate cancer patients: A critical review. Can Urol Assoc J 6(2): 121-127, 2012.
36 Wynants L, Bouwmeester W, Moons KGM, Moerbeek M, Timmerman D, Van Huffel S, Van Calster B and Vergouwe Y: A simulation study of sample size demonstrated the importance of the number of events per variable to develop prediction models in clustered data. J Clin Epidemiol 68(12): 1406-1414, 2015.

37 Saw CB, Chen H and Wagner JH: Implementation of fiducialbased image registration in the cyberknife robotic system. Med Dosim 33: 156-160, 2008.

38 Laverdiere J, Nabid A, De Bedoya LD, Ebacher A, Fortin A, Wang CS and Harel F: The efficacy and sequencing of a short course of androgen suppression on freedom from biochemical failure when administered with radiation therapy for T2-T3 prostate cancer. J Urol 171(3): 1137-1140, 2004.
Received November 30, 2017

Revised January 8, 2018

Accepted January 11, 2018 